\title{
Bioethanol Transformations Over Active Surface Sites Generated on Carbon Nanotubes or Carbon Nanofibers Materials
}

\author{
M. Almohalla ${ }^{1}$, M.V. Morales ${ }^{1}$, E. Asedegbega-Nieto ${ }^{1}$, A. Maroto-Valiente ${ }^{1}$, B. Bachiller-Baeza ${ }^{2}$, \\ I. Rodríguez-Ramos ${ }^{2}$ and A. Guerrero-Ruiz ${ }^{*}$
}

\author{
${ }^{I}$ Dpto. Química Inorgánica y Química Técnica, Facultad de Ciencias, UNED, Madrid, Spain \\ ${ }^{2}$ Instituto de Catálisis y Petroleoquímica, CSIC, Campus Cantoblanco Madrid, Spain
}

\begin{abstract}
Catalytic bioethanol transformations over carbon nanomaterials (nanofibers and nanotubes) have been evaluated at atmospheric pressure and in the temperature range of 473-773 K. The pristine carbon materials were compared with these samples after surface modification by introducing sulfonic groups. The specific activity for ethanol dehydrogenation, yielding acetaldehyde, increases with the surface graphitization degree for these materials. This suggests that some basic sites can be related with specific surface graphitic structures or with the conjugated basic sites produced after removing acidic oxygen surface groups. Concerning the dehydration reaction over sulfonated samples, it is observed that catalytic activities are related with the amount of incorporated sulfur species, as detected by the evolution of $\mathrm{SO}_{2}$ in the Temperature programmed Desorption (TPD) as well as by the analysis of sulfur by X-Ray Photoelectron Spectroscopy (XPS).
\end{abstract}

Keywords: Acid-basic properties, bioethanol, carbon nanomaterials, dehydration catalysts, dehydrogenation.

\section{INTRODUCTION}

The utilization of biomass for the production of fuels and chemicals is currently an area of great activity because of the recognition that fossil fuels are a finite resource and because of the imminent threat of global warming from the release of carbon dioxide [1-6]. Among the major products of biomass conversion is ethanol, a commodity chemically derived from the fermentation of sugarcane or energy-rich crops such as corn $[7,8]$. Although ethanol is produced in large quantities as a substitute or supplement for fuels derived from crude oil, it is currently unlikely to replace much less expensive chemical feedstocks derived from petroleum or natural gas such as ethylene. Nevertheless, in a future with limited hydrocarbon supplies, there may be a role for ethanol as a primary feedstock [9] as recently demonstrated by the startup of a 200,000 metric ton ethylene from ethanol plant in Brazil [10], and for this reason, research in ethanol conversion is warranted. It is also of interest to study ethanol for fundamental reasons. First, it is one of the simplest oxygenated compounds, and its deoxygenation can provide insights into oxygen removal from more complicated molecules. Second, it can react to produce different products and for this reason can be used as a probe to relate the reaction pathway to the properties of the catalysts, in particular their acid/base properties.

The more feasible transformations of ethanol by heterogeneous catalysts are those yielding ethylene or

*Address correspondence to this author at the Dpto. Química Inorgánica y Química Técnica, Facultad de Ciencias, UNED, Madrid, Spain;

Tel: 34913987344; Fax: 34913986697; E-mail: aguerrero@ccia.uned.es acetaldehyde. In the first case ethanol is transformed by dehydration over acid surface sites [11-13], while acetaldehyde is obtained by dehydrogenation over basic sites $[14,15]$.

As it is known, carbon materials catalyze dehydration and/or dehydrogenation of aliphatic alcohols [16-20]. The decomposition of ethanol on solid catalysts typically occurs through competing reactions [8, 10, 21]: (1) intermolecular dehydration, which gives diethyl ether (DEE) and water; (2) intramolecular dehydration, which yields ethylene and water; (3) dehydrogenation, which produces acetaldehyde and hydrogen; and (4) total decomposition into $\mathrm{CO}, \mathrm{H}_{2}, \mathrm{CH}_{4}, \mathrm{C}$, and $\mathrm{O}$ [7]. The acidity and basicity of the solid catalysts are important factors that influence their activity and selectivity.

An important pathway for ethanol conversion is dehydration to ethylene. Previous studies reported that HZSM-5 zeolite with strong Brønsted acid sites was an effective catalyst for this transformation [22]. However, deactivation due to coke formation on its surface led to decreasing activity and selectivity towards ethylene [23] and therefore made the process unsuitable for industrial applications [24]. Phillips and Datta [25] investigated the production of ethylene from hydrous ethanol over HZSM-5 under mild conditions and demonstrated that strong Brønsted acid sites led to rapid catalyst deactivation in the initial stages through the oligomerization of ethylene and the formation of carbonaceous species. However, moderating the acidity could reduce coke formation and enhance the steadystate catalytic activity of HZSM-5. Another important pathway for ethanol decomposition is dehydrogenation to acetaldehyde and hydrogen, which is favored on basic [26] or metallic catalysts [27]. Chang et al. reported that copper 
catalysts supported on rice husk ash displayed high catalytic activity and selectivity towards dehydrogenation products [27]. Much attention has been focused on preparing bifunctional catalysts with both acidic and basic sites in order to promote both dehydrogenation and dehydration reactions [28-31]. Less effort has been focused on the study of ethanol dehydration over carbon based catalysts, including nanocarbons (nanotubes, nanofibers, etc). The few papers studying carbon catalysts employ additional surface treatments with different oxidants, such as $\left(\mathrm{NH}_{4}\right)_{2} \mathrm{~S}_{2} \mathrm{O}_{8}$ [32] y $\mathrm{HNO}_{3}$ [33], to generate acid surface groups, as carboxyl acid groups, of low to moderate thermal stability.

The objective of this work is to study the preparation of modified carbon nanomaterials with different surface chemical properties and to analyze their catalytic behavior in the decomposition of ethanol. We find catalytic performance, mainly ascribable to basic surface sites, for 4 carbon catalytic materials. These are: two commercial nanofibers from Pyrograph products Inc (with different degree of surface graphitization) and two multiwall carbon nanotubes, one of them provided by Nanocyl and the other lab prepared-treated. These four carbon materials were also further treated with fuming sulfuric acid, in order to incorporate sulfonic groups, and the resulting acid catalyst samples were tested in the dehydration of bioethanol taking advantage of their acidic properties.

\section{EXPERIMENTAL}

\subsection{Materials}

Commercial Carbon nanofibers, Pyrograph III PR24HHT (CNFs-HHT $\mathrm{S}_{\mathrm{BET}}=32 \mathrm{~m}^{2} / \mathrm{g}$ ), provided by Applied Sciences Inc., were originally treated at high temperature $(\sim 3273 \mathrm{~K})$. These fibers possess a stacked-cup morphology with a hollow core through the length of the fiber and also present a jagged outer surface with "round heads" or "loop" structures which connect several layers [34, 35]. Commercial carbon nanofibers Pyrograph III, PR24-PS (CNFs-PS $\mathrm{S}_{\mathrm{BET}}=$ $36 \mathrm{~m}^{2} / \mathrm{g}$ ), are pyrolitically stripped carbon fiber at $1373 \mathrm{~K}$, and therefore, less graphitized than CNF-HHT. Multiwall carbon nanotubes, (MWCNTs $\mathrm{S}_{\mathrm{BET}}=84 \mathrm{~m}^{2} / \mathrm{g}$ ) synthesized by the floating catalyst method [36], and subsequently treated with concentrated $\mathrm{HNO}_{3}(65 \%)\left(\right.$ MWCNTs-ox, $\left.\mathrm{S}_{\mathrm{BET}}=96\right)$ as described elsewhere [37]. Commercial multiwall carbon nanotubes (CNTs-Nanocyl, $\mathrm{S}_{\mathrm{BET}}=360 \mathrm{~m}^{2} / \mathrm{g}$ ) were provided by NanocylTM 3100 Series, $95 \%$ purity.

\subsection{Sulfonation of Carbon Materials}

Aliquots of $3 \mathrm{~g}$ of carbon nanomaterials were immersed in $150 \mathrm{~mL}$ of fuming sulfuric acid $\left(15 \mathrm{wt} \% \mathrm{SO}_{3}\right)$ at $353 \mathrm{~K}$ under a nitrogen gas atmosphere for $7 \mathrm{~h}$. After the treatment, the suspension was washed with deionized water to remove any excess sulfuric acid in the carbon nanomaterial, filtered and dried at $393 \mathrm{~K}$.

\subsection{Samples Characterization}

The nature and relative concentrations of sulfonic groups were evaluated by X-ray photoelectron spectroscopy (XPS). $\mathrm{X}$-ray photoelectron spectra were recorded with an Omicron spectrometer equipped with an EA-125 hemispherical electron multichannel analyzer and an unmonochromatized $\mathrm{Mg} \mathrm{K} \alpha \mathrm{X}$-ray source having radiation energy of $1253.6 \mathrm{eV}$ at
$150 \mathrm{~W}$ and a pass energy of $50 \mathrm{eV}$. The samples $(0.2 \mathrm{mg})$ were slightly pressed into small pellets of $15 \mathrm{~mm}$ diameter and then mounted on the sample holder. Every sample was introduced into the chamber where they were degassed for 6$8 \mathrm{~h}$, in order to achieve a dynamic vacuum below $10^{-8} \mathrm{~Pa}$ prior to analysis. Spectra were analyzed with CasaXPS software and RSF database by fitting after Shirley background correction.

The chemical nature of these functional groups was also evaluated by temperature programmed desorption. These experiments (TPD-MS) were performed under vacuum, in a conventional volumetric apparatus connected to a SRS RGA-200 mass spectrometer. The sample was evacuated for $30 \mathrm{~min}$ at room temperature and then ramped to $1023 \mathrm{~K}$ at a $10 \mathrm{~K} / \mathrm{min}$ rate.

Specific surface areas were calculated using the BET method from nitrogen adsorption isotherms, recorded at the temperature of liquid nitrogen on a Micromeritics ASAP 2010 apparatus.

Elemental chemical analysis of carbon, hydrogen, nitrogen and sulfur was carried out in a LECO CHNS-932 instrument.

\subsection{Ethanol Decomposition}

The catalytic activity of the carbon nanomaterials was studied in the decomposition of ethanol in gas phase at atmospheric pressure (PID Microactivity) in a fixed bed microreactor (i.d. $0.9 \mathrm{~mm}$ ). In a typical experiment $200 \mathrm{mg}$ of catalysts $(0.5-1 \mathrm{~mm})$ were dispersed in solid-glass beads, in order to increase the bed length and avoid local heating. Ethanol was charged through a micro pump, using helium as carrier gas and working at atmospheric pressure. The total flow rate of the vapor stream was kept constant at 20.66 $\mathrm{mL} / \mathrm{min}$. To avoid the condensation of any reactant or product, all the pipelines were heated up to $423 \mathrm{~K}$. The compositions of the reactor effluent streams were analyzed using a gas chromatograph (Varian CP-3800) equipped with a Porapak Q column and an FID detector. Blank experiments were done to verify the absence of catalytic activity, in the conditions used in this study, either with the empty reactor or filled with the glass beads.

The conversion was defined as the ratio of the amount of ethanol converted to the amount of ethanol supplied to the reactor. The selectivity (in mol\%) was defined as the molar ratio of a specific product to all the products formed.

\section{RESULTS AND DISCUSSION}

\subsection{Quantification of Functional Groups on the Nanomaterials Surface}

The XPS technique was used in this current work to determine the nature and relative abundance of functional groups present on the nanomaterial surfaces. The S2p spectra (Fig. 1a) corresponding to S-functionalized samples reveal a binding energy of $169 \mathrm{eV}$, characteristic of $-\mathrm{SO}_{3} \mathrm{H}$ groups with sulfur in its +6 state [38]. In the Fig. (1b), the S2p spectra of the $\mathrm{S}$ functionalized samples after use are also shown. In this case, the CNF-HHT sample after reaction displayed a second S2p peak at $163.9 \mathrm{eV}$, which is assigned in the literature to reduced forms of sulfur [39, 40]. This demonstrates that all the sulfur in the sulfonated samples, 
before the reaction, can be associated to sulfonic groups. Quantitative S/C atomic ratios and C 1s, O 1s y S 2p (\%) atomic concentrations after the fuming sulfuric acid treatments are shown in Table 1. It is worth noting that the sample CNF-PS-SO ${ }_{3}$ showed the highest $\mathrm{S} / \mathrm{C}$ surface atomic ratio $(0.03 \%)$. From the surface oxygen $(\mathrm{O} 1 \mathrm{~s})$ percentages presented in Table 1, it appears that these are more than three times that of $\mathrm{S} 2 \mathrm{p}$. Thus apart from $\mathrm{SO}_{3}$ groups some additional oxygen surface groups could be incorporated on all the carbon nanomaterials with the exception of the MWCNTs-ox- $\mathrm{SO}_{3}$ because the non-sulfated sample already has an important presence of surface oxygen groups (Table 1). These acid groups are also apparent in the C1s peak, which can be mainly quantified as carboxyls or carbonyls species by deconvolution.

The total S/C content of samples was determined by means of elemental analysis (Table 1), which is in good agreement with the results obtained by XPS.

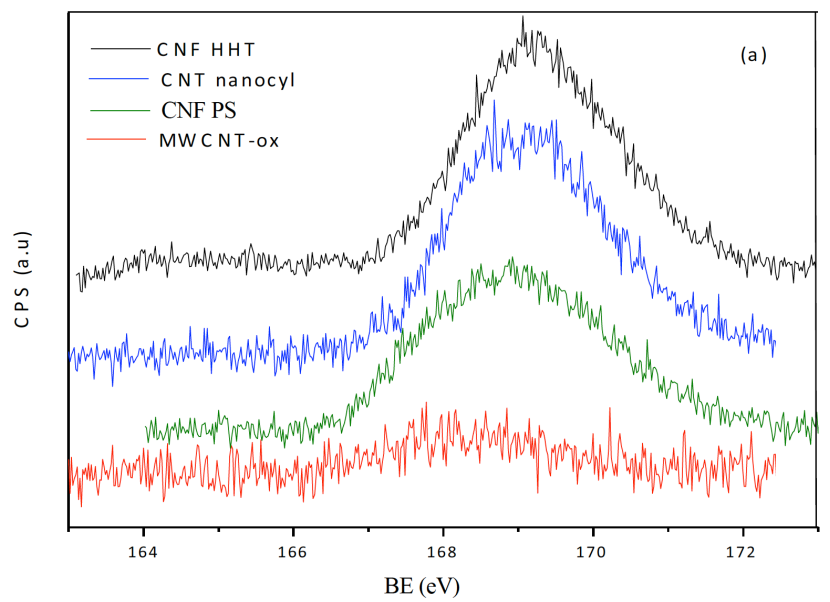

In order to complement these analyses, temperatureprogrammed desorption experiments were carried out. The TPD-MS profiles of the different samples are compiled in Fig. (2). The fragments $\mathrm{m} / \mathrm{e}=28,44,2,18$ and 64 corresponding to $\mathrm{CO}, \mathrm{CO}_{2}, \mathrm{H}_{2}, \mathrm{O}_{2}$ and $\mathrm{SO}_{2}$, respectively, were monitored. Analyzing the $\mathrm{CO}$ and $\mathrm{CO}_{2}$ spectra, it is evident that the treatment with fuming acid introduces oxygen functional groups on the surface of the materials, which decompose as $\mathrm{CO}$ and $\mathrm{CO}_{2}$ upon heating. These correspond mainly to phenols/quinones and carboxylic acids, which are decomposed as $\mathrm{CO}$ above $873 \mathrm{~K}$ and as $\mathrm{CO}_{2}$ between 403 and $723 \mathrm{~K}$, respectively $[40,41]$. The analysis of the $\mathrm{SO}_{2}$ spectra reveals that all materials treated with sulfuric acid contain sulfur functional groups, the higher fraction desorbing between 623$663 \mathrm{~K}$ is associated with sulfonic acids groups [42, 43], which is in agreement with XPS spectra of S2p (Fig. 1). The evolution $\mathrm{SO}_{2}$ peaks are clearly highest for CNF-PS, indicating that this sample contains the maximum S content, and this is confirmed by the XPS results shown in Table 1.

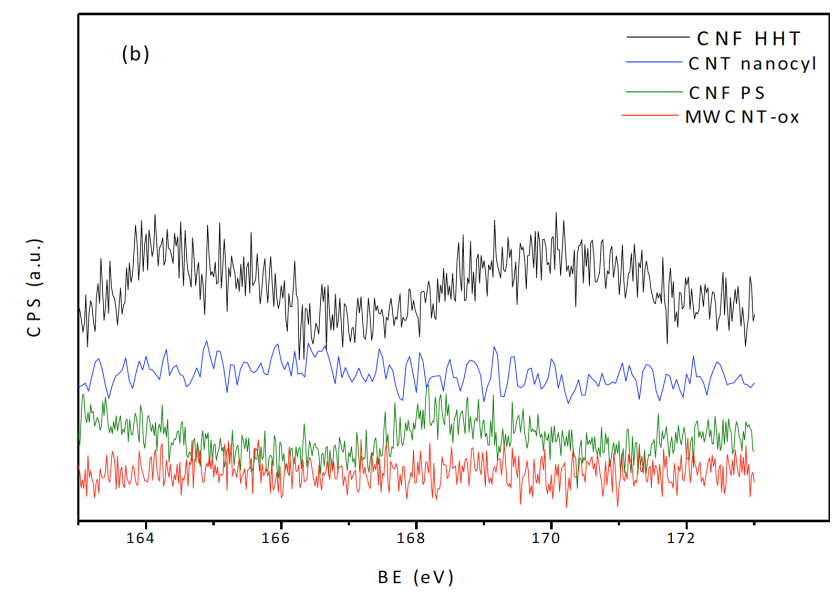

Fig. (1). S 2p XPS spectra of fuming acid treated nanomaterials (a) before reaction, and (b) after reaction.

Table 1. Surface Composition Determined by XPS

\begin{tabular}{|c|c|c|c|c|}
\hline \multicolumn{5}{|l|}{ Non Sulfonated Samples } \\
\hline \multicolumn{5}{|c|}{$\%$ Atomic Concentration } \\
\hline & CNF-HHT & CNF-PS & CNT- nanocyl & MWCNT-ox \\
\hline $\mathrm{C} 1 \mathrm{~s}$ & 99.30 & 97.90 & 99.39 & 94.67 \\
\hline $\mathrm{O} 1 \mathrm{~s}$ & 0.70 & 2.09 & 0.61 & 5.33 \\
\hline S2p & 0.00 & 0.00 & 0.00 & 0.00 \\
\hline \multicolumn{5}{|l|}{ Sulfonated Samples } \\
\hline \multicolumn{5}{|c|}{$\%$ Atomic Concentration } \\
\hline & CNF-HHT-SO & $\mathrm{CNF}_{-\mathrm{PS}-\mathrm{SO}_{3}}$ & CNTs-nanocyl-SO $_{3}$ & MWCNTs-ox-SO \\
\hline $\mathrm{C} 1 \mathrm{~s}$ & 90.19 & 81.74 & 92.12 & 89.99 \\
\hline $\mathrm{O} 1 \mathrm{~s}$ & 8.48 & 14.57 & 6.57 & 10.87 \\
\hline S2p & 1.34 & 2.68 & 1.30 & 1.42 \\
\hline $\mathrm{S} / \mathrm{C}$ & 0.01 & 0.03 & 0.01 & 0.01 \\
\hline $\mathrm{S} / \mathrm{C}$ Elemental analysis & 0.0041 & 0.0065 & 0.0052 & - \\
\hline
\end{tabular}



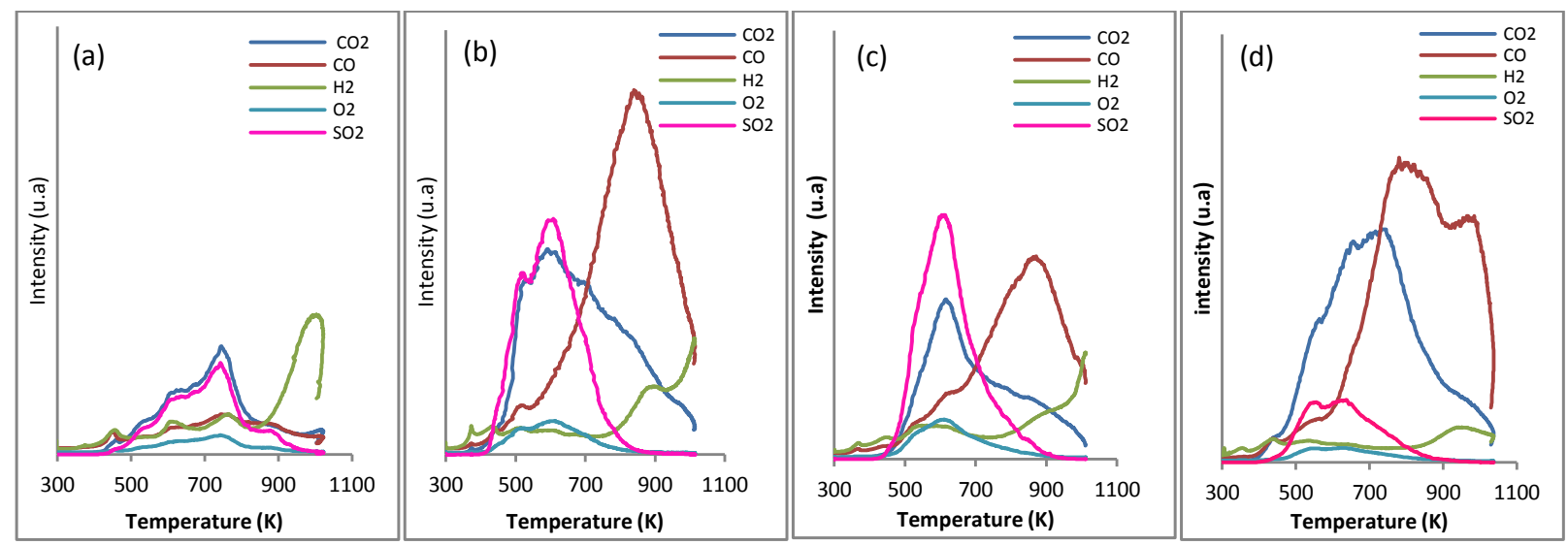

Fig. (2). TPD spectra of (a) CNF-HHT-SO $;$; (b) CNF-PS-SO 3 ; (c) CNT-nanocyl-SO $\mathrm{SO}_{3}$; (d) $\mathrm{MWCNT}-\mathrm{ox}-\mathrm{SO}_{3}$.

\subsection{Catalytic Activity}

Catalytic activities of the nanomaterials treated with fuming acid were tested in vapor phase ethanol dehydration reaction, while non-treated nanomaterials mainly produce ethanol dehydrogenation reaction. These experiments were performed in a fixed bed reactor and studied in a wide range of operating temperatures, since the product distribution of ethanol catalytic decomposition strongly depends on reaction temperatures. For each catalyst these experiments were performed twice, in order to check the reproducibility of these determinations. The data points reported here correspond to the average of these two measurements.

As shown in Table 2, the non-treated catalysts showed higher activity in the dehydrogenation reaction, with acetaldehyde selectivity reaching $100 \%$ (Fig. 3). The gradual change of product selectivity particularly for CNT-nanocyl and MWCNTs corresponds with the evolution of the surface chemistry properties of the samples with the increasing temperature, as proved by TPD and not shown here for the sake of brevity. The MWCNTs sample shows the most drastic change in selectivity from $80 \%$ DEE at $473 \mathrm{~K}$ to $92 \%$ acetaldehyde at $723 \mathrm{~K}$. This sample has the highest content of surface oxygen group (XPS data) and with acid character (TPD profiles) which catalyzed the dehydratation of ethanol to DEE. Evolution of these carboxylic groups with the increasing reaction temperature leads to products catalyzed by the free (clean) graphitic surface. Comparison of catalytic activities at $723 \mathrm{~K}$ reaction temperature for the different nontreated carbon materials reveals that it seems to be higher for those with larger surface area. However, when the catalytic activity is expressed as ethanol micromoles converted per second and per square meter it is seen that the CNF-HHT and MWCNT-ox samples present the highest values. The studies by Raman spectroscopy $[35,44,45]$ indicate that the intensity ratio of $D$ to $G$ bands $\left(\mathrm{I}_{\mathrm{D}} / \mathrm{I}_{\mathrm{G}}\right)$, which can be related to the degree of the graphitic disorder, is lower for these two samples (Table 2). This suggests that basic surface sites can be related with graphitic structures which are active and selective for the acetaldehyde formation. In the second case, it should be considered that acid surface groups are removed during reaction operating at relatively moderate temperatures $(473-673 \mathrm{~K})$ and the resulting surface at $723 \mathrm{~K}$ has basic character. Therefore, the elimination of oxygen surface

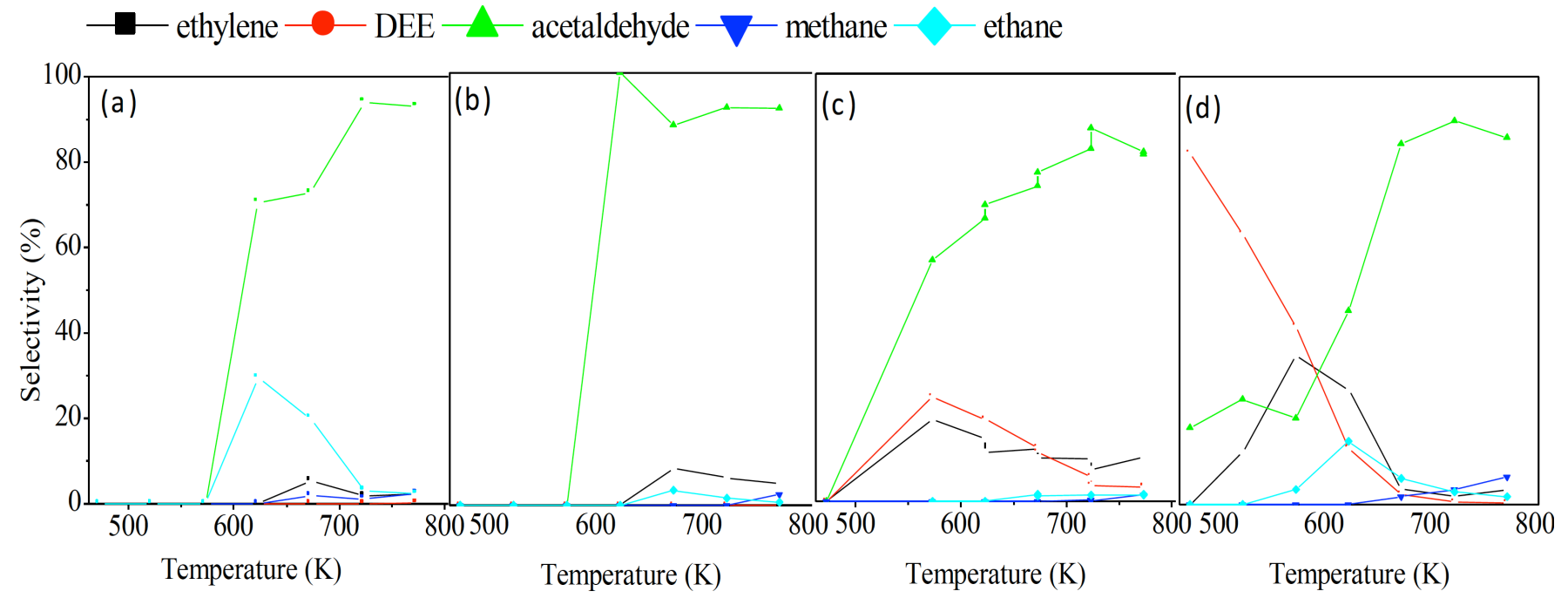

Fig. (3). Selectivity towards products in the dehydration of ethanol over functionalized nanomaterials (a) CNF-HHT, (b) CNF-PS (c) CNTnanocyl, and (d) MWCNTS-ox. 
Table 2. Catalytic Results of Dehydrogenation Ethanol (723 K)

\begin{tabular}{|c|c|c|c|c|c|}
\hline Catalyst & $\mathrm{S}_{\text {BET }}$ & $\mathbf{I}_{\mathbf{D}} / \mathbf{I}_{\mathrm{G}} *$ & Activity ( $\mu \mathrm{mol}$ conv/g.s) & Specific Activity ( $\mu$ molconv $\left./ \mathrm{m}^{2} . \mathrm{s}\right)$ & S (\%) Acetaldehyde \\
\hline CNF-HHT & 32 & 0.14 & 1.55 & 0.05 & 94 \\
\hline CNF-PS & 36 & 1.80 & 0.26 & 0.01 & 91 \\
\hline CNT-nanocyl & 331 & 2.1 & 6.48 & 0.02 & 82 \\
\hline MWCNTs-ox & 96 & 0.95 & 4.13 & 0.04 & 92 \\
\hline
\end{tabular}

*Data from references $[43,44]$.

$\longrightarrow$ ethylene - DEE $-\square-$ acetaldehyde $-\square$ methane - ethane

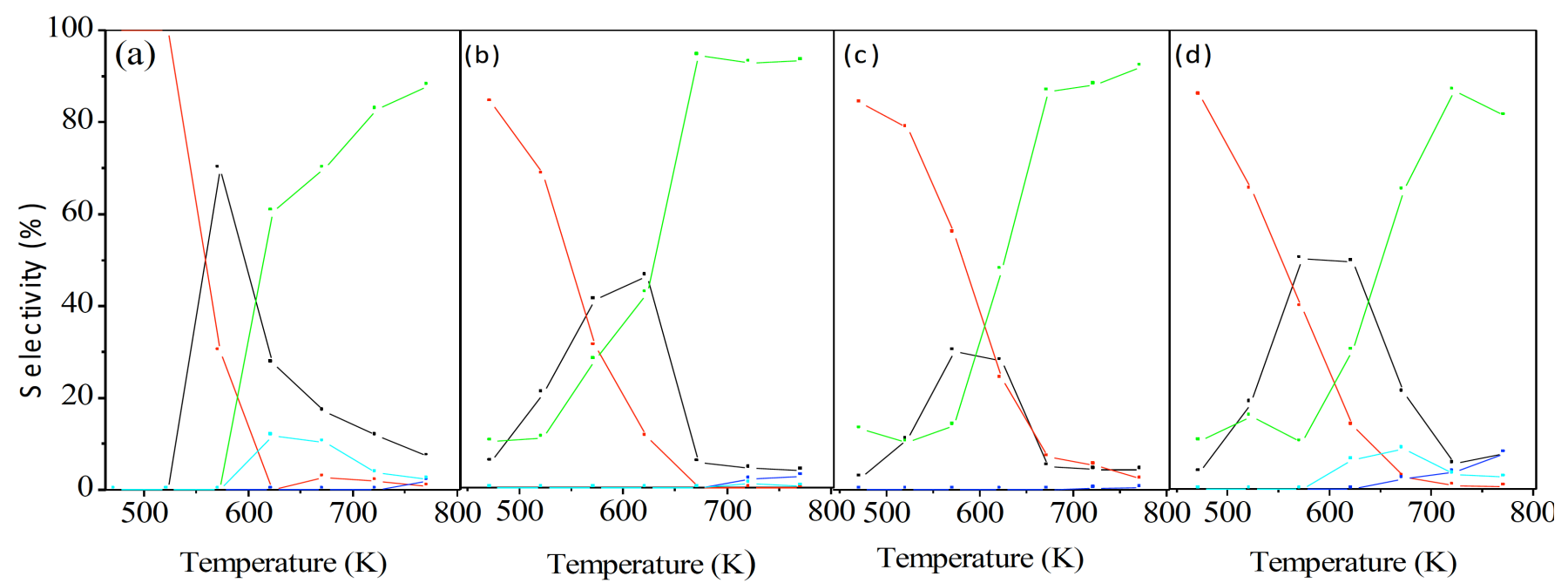

Fig. (4). Selectivity towards products in the dehydration of ethanol over functionalized nanomaterials (a) $\mathrm{CNF}-\mathrm{HHT}_{-} \mathrm{SO}_{3},(\mathbf{b}) \mathrm{CNF}^{-\mathrm{PS}-\mathrm{SO}_{3}}$ (c) CNT-nanocyl-SO $\mathrm{SO}_{3}$, and (d) MWCNTS-ox- $\mathrm{SO}_{3}$.

groups exposes the bulk graphitic structures detected by Raman in this sample.

Concerning the sulfonated samples, the modification of the chemical nature of the carbon surface by sulfonic group incorporation is accompanied by changes in the product selectivity, increasing the dehydration selectivity. As shown in Fig. (4), at lower temperatures, the dehydration of ethanol is predominant, being the two main products ethylene and DEE, while at temperatures higher than $623 \mathrm{~K}$, dehydrogenation is predominant with the formation of acetaldehyde. Ethylene and DEE are formed due to dehydration reactions taking place on the acid sites of the catalyst [46]. However, acetaldehyde formation through the dehydrogenation reaction reveals the presence of basic sites exposed once sulfonated groups disappeared.

Table 3. Catalytic Results of Dehydration Ethanol (573 K)

\begin{tabular}{|c|c|c|c|c|c|c|}
\hline Catalyst & $\mathbf{S}_{\text {BET }}$ & Activity $(\boldsymbol{\mu m o l ~ c o n v / s )}$ & Specific Activity $\left(\boldsymbol{\mu m o l ~ c o n v / \mathbf { m } ^ { 2 } . \mathbf { s } )}\right.$ & S(\%) Ethylene & S(\%) DEE & S(\%) Acetaldehyde \\
\hline \hline CNF-HHT-SO $_{3}$ & 31 & 0.08 & 0.001 & 69.86 & 30.14 & $\mathrm{nd}^{*}$ \\
\hline CNF-PS-SO $_{3}$ & 37 & 0.44 & 0.021 & 41.08 & 30.93 & 27.99 \\
\hline CNT-nanocyl-SO $_{3}$ & 252 & 0.60 & 0.002 & 50.09 & 35.11 & 14.08 \\
\hline MWCNTs-ox-SO $_{3}$ & 124 & 0.26 & 0.010 & 50.16 & 39.65 & 10.18 \\
\hline
\end{tabular}

As shown in Fig. (4), the maximum of DEE selectivity is achieved close to $473 \mathrm{~K}$, for all catalysts. Further increase in reaction temperature produces a decline in the DEE selectivity in favor of that of ethylene. In agreement with previous results with further increase in reaction temperature, DEE is expected to decompose, producing more ethylene [47-49]. It was quite interesting to note that as the sulfonic groups are decomposed the graphitic surfaces catalyze the dehydrogenation reaction.

Table 3 summarizes the catalytic data of all modified catalysts in the decomposition of ethanol at $573 \mathrm{~K}$. This is the temperature at which the dehydration reaction activity is highest. It should be noted that the catalyst with the highest specific activity for the ethanol dehydration is CNF-PS-SO In these cases the catalytic activities can be related with the area of $\mathrm{SO}_{2}$ evolved during TPD (Fig. 2), as well as with the 
amount of S detected in XPS analysis. Thus, sample CNF$\mathrm{PS}-\mathrm{SO}_{3}$ that is initially more reactive, is where highest amounts of incorporated sulfur were detected, and thus becomes the most active one. Finally it should be remarked that the thermal stabilities of these samples is very limited, and so regeneration seems not to be feasible.

\section{CONCLUSION}

Dehydrogenation of ethanol over a series of carbon nanomaterials and ethanol dehydration to ethylene over sulfur modified nanocarbons, were studied in this work. In this latter case TPD profiles of modified samples show that the $\mathrm{SO}_{2}$ peak corresponding to sulfonic group decomposition is clearly higher for CNF-PS-SO ${ }_{3}$, indicating that this sample contains the maximum $\mathrm{S}$ content. This is in agreement with elemental analysis determinations and XPS results, as XPS analysis of this sample reveals the highest \% $\mathrm{S} / \mathrm{C}$ surface atomic ratio.

Under the experimental conditions of this work, we found higher catalytic activity, in the dehydrogenation reaction at $723 \mathrm{~K}$, for non-treated carbon nanomaterials with higher graphitization degree as revealed by Raman spectroscopy. The modification of the chemical nature of the carbon surface, introducing sulfonic acid groups, changes the product selectivity. It increases the dehydration selectivity, leading to diethyl ether, predominating at temperatures of $473-523 \mathrm{~K}$ and to ethylene predominating at temperatures of 573-623 K. In the case of materials treated with sulfuric acid this can be related to the presence of acid surface sites. However these acidic species are not stable enough under reaction conditions. As revealed by XPS the sulfur content decreases for the used samples.

Thus, it can be concluded that carbon nanomaterials are promising for the preparation of "ecofriendly" catalysts, both for the dehydrogenation of bioethanol to yield acetaldehyde or for ethylene production by dehydration of ethanol. Although in this latter case improvement in the stabilization of sulfur surface species is required.

\section{CONFLICT OF INTEREST}

The authors confirm that this article content has no conflict of interest.

\section{ACKNOWLEDGEMENTS}

The financial support of the Spanish government by Projects CTQ2011-29272-C04-01 and 03 is recognized.

\section{REFERENCES}

[1] Choudhary, T.V.; Phillips, C.B. Renewable fuels via catalytic hydrodeoxygenation. Appl. Catal., A: Gen., 2011, 397, 1-12.

[2] Zakzeski, J.; Bruijnincx, P.C.A.; Jongerius, A.L.; Weckhuysen, B.M. The catalytic valorization of lignin for the production of renewable chemicals. Chem. Rev., 2010, 110, 552-3599.

[3] Wawrzetz, A.; Peng, B.; Hrabar, A.; Jentys, A.; Lemonidou, A.A.; Lercher, J.A. Towards understanding the bifunctional hydrodeoxygenation and aqueous phase reforming of glycerol. $J$. Catal., 2010, 269, 411-420.

[4] Casanova, O.; Iborra, S.; Corma, A. Chemicals from biomass: Etherification of 5-hydroxymethyl-2-furfural (HMF) into 5,5 '(oxybis(methylene))bis-2-furfural (OBMF) with solid catalysts. $J$. Catal., 2010, 275, 236-242.

[5] Bozell, J.J.; Petersen, G.R. Technology development for the production of biobased products from biorefinery carbohydrates- the US Department of Energy's "Top 10" revisited. Green Chem., 2010, 12, 539-554.

[6] Serrano-Ruiz, J.C.; Wang, D.; Dumesic, J.A. Catalytic upgrading of levulinic acid to 5-nonanone. Green Chem., 2010, 12, 574-577.

[7] Birot, A.; Epron, F.; Descorme, C.; Duprez, D. Ethanol steam reforming over $\mathrm{Rh} / \mathrm{Ce}_{\mathrm{x}} \mathrm{Zr}_{1-\mathrm{x}} \mathrm{O}_{2}$ catalysts: Impact of the $\mathrm{CO}-\mathrm{CO}_{2-}$ $\mathrm{CH}_{4}$ interconversion reactions on the $\mathrm{H}_{2}$ production. Appl. Catal. B-Environ., 2008, 79, 17-25.

[8] Rass-Hansen, J.; Johansson, R.; Moller, M.; Christensen, C.H. Steam reforming of technical bioethanol for hydrogen production. Int. J. Hydrogen Energy, 2008, 33, 4547-4554.

[9] Pereira, E.B.; de la Piscina, P.R.; Homs, N. Efficient hydrogen production from ethanol and glycerol by vapour-phase reforming processes with new cobalt-based catalysts. Bioresour. Technol., 2011, 102, 3419-3423.

[10] Tullo, A.H. Braskem's Push. Chem. Eng. News, 2011, 89, $24-25$.

[11] El-Katatny, E.A.; Halawy, S.A.; Mohamed, M.A.; Zaki, M.I. Recovery of ethene-selective $\mathrm{FeO}_{\mathrm{x}} / \mathrm{Al}_{2} \mathrm{O}_{3}$ ethanol dehydration catalyst from industrial chemical wastes. Appl. Catal. A: Gen., 2000, 199, 83-92.

[12] Machado, N.R.C.F.; Calsavara, V.; Astrath, N.G.C.; Matsuda, C.K.; Junior, A.P.; Baesso, M.L. Obtaining hydrocarbons from ethanol over iron-modified ZSM-5 zeolites. Fuel, 2005, 84, 20642070.

[13] Calsavara, V.; Baesso, M.L.; Machado, N.R.C.F. Transformation of ethanol into hydrocarbons on ZSM-5 zeolites modified with iron in different ways. Fuel, 2008, 87, 1628-1636.

[14] Takezawa, N.; Hanamaki, C.; Kobayashi, H. Mechanism of dehydrogenation of ethanol on magnesium oxide. J. Catal., 1975, 38, 101-109.

[15] Matsumura, Y.; Hashimoto, K.; Yoshida, S. Selective dehydrogenation of ethanol over highly dehydrated silica. J. Catal., 1989, 117, 135-143.

[16] Tsuruizumi, A. Porous structure of active carbons.10. Dehydration of alcohols in presence of active carbons.1. Nippon Kagaku Zasshi, 1961, 82-5, 545-550.

[17] Tsuruizumi, A. Dehydration of alcohols in presence of active carbon. 2. Nippon Kagaku Zasshi, 1961, 82-9, 1111-1119.

[18] Szymanski, G.S.; Rychlicki, G. Catalytic conversion of 2-propanol on cation-substituted froms of oxidized carbon. React. Kinet. Catal. Lett., 1991, 43, 475-479.

[19] Szymasnki, G. S.; Rychlicki, G. Importance of oxygen-surface groups in catalytic dehydration and dehydrogenation of butan-2-ol promoted by carbon catalytsts. Carbon, 1991, 29, 489-498.

[20] Szymasnki, G. S.; Rychlicki, G. Catalytic conversion of propan-2ol on carbon catalysys. Carbon, 1993, 31, 247-257.

[21] Guenard, R.L.; Fernandez-Torres, L.C.; Kim, B.I.; Perry, S.S.; Frantz, P.; Didziulis, S.V. Selective surface reactions of single crystal metal carbides: alkene production from short chain alcohols on titanium carbide and vanadium carbide. Surf. Sci., 2002, 515, 103-116.

[22] Takahara, I.; Saito, M.; Inaba, M.; Murata, K. Dehydration of ethanol into ethylene over solid acid catalysts. Catal. Lett., 2005, 105, 249-252.

[23] Zhang, X.; Wang, R.; Yang, X.; Zhang, F. Comparison of four catalysts in the catalytic dehydration of ethanol to ethylene. Micropor. Mesopor. Mater., 2008, 116, 210-215.

[24] Schulz, J.; Bandermann, F. Conversion of ethanol over zeolite HZSM-5. Chem. Eng. Technol., 1994, 17, 179-186.

[25] Phillips, C.B.; Datta, R. Production of ethylene from hydrous ethanol on H-ZSM-5 under mild conditions. Ind. Eng. Chem. Res., 1997, 36, 4466-4475.

[26] Vohs, J.M.; Barteau, M.A. Dehydration and dehydrogenation of ethanol and 1-propanol on the polar surfaces of zinc oxide. Surf. Sci., 1989, 211, 590-608.

[27] Chang, F.W.; Kuo, W.Y.; Lee, K.C. Dehydrogenation of ethanol over copper catalysts on rice husk ash prepared by incipient wetness impregnation. Appl. Catal. A: Gen., 2003, 246, 253-264.

[28] Aramendía, M.A.; Borau, V.; Jiménez, C.; Marinas, J.M.; Porras, A.; Urbano, F.J. Magnesium oxides as basic catalysts for organic processes study of the dehydrogenation-dehydration of 2-propanol. J. Catal., 1996, 161, 829-838.

[29] Shinohara, Y.; Nakajima, T.; Suzuki, S. A theoretical study of the dehydration and the dehydrogenation processes of alcohols on metal oxides using MOPAC. J. Mol. Struc. Theochem., 1999, 460, 231-244. 
[30] Doheim, M.M.; El-Shobaky, H.G. Catalytic conversion of ethanol and iso-propanol over $\mathrm{ZnO}$-treated $\mathrm{Co}_{3} \mathrm{O}_{4} / \mathrm{Al}_{2} \mathrm{O}_{3}$ solids. Colloids Surf. A, 2002, 204, 169-174.

[31] Ramos, F.S.; Duarte de Farias, A.M.; Borges, L.E.P.; Monteiro, J.L.; Fraga, M.A.; Sousa-Aguiar, E.F.; Appel, L.G. Role of dehydration catalyst acid properties on one-step DME synthesis over physical mixtures. Catal. Today, 2005, 101, 39-44.

[32] Carrasco-Marín, F.; Mueden, A.; Moreno-Castilla, C. Surfacetreated activated carbons as catalysts for the dehydration and dehydrogenation reactions of ethanol. J. Phys. Chem. B, 1998, 102, 9239-9244.

[33] Szymanski, G.S.; Rychlicki, G.; Terzyk, A.P. Catalytic conversion of ethanol on carbon catalysts. Carbon, 1994, 32, 265-271.

[34] Asedegbega-Nieto, E.; Bachiller-Baeza, B.; Kuvshinov, D.G.; Garcia-Garcia, F.; Chukanov, E.; Kushinov, G.G.; Guerrero-Ruiz, A.; Rodriguez-Ramos, I. Effect of the carbon support nanostructures on the performance of Ru catalysts in the hydrogenation of paracetamol. Carbon, 2008, 46, 1046-1052.

[35] Dongil, A.B.; Bachiller-Baeza, B.; Guerrero-Ruiz, A.; RodriguezRamos, I.; Martinez-Alonso, A.; Tascon. J.M.D. Surface chemical modifications induced on high surface area graphite and carbon nanofibers using different oxidation and functionalization treatments. J. Colloid Interf. Sci., 2011, 355, 179-189.

[36] Sampedro-Tejedor, P.; Maroto-Valiente, A.; Nevskaia, D.M.; Muñoz, V.; Rodriguez-Ramos, I.; Guerrero Ruiz, A. The effect of growth temperature and iron precursor on the synthesis of high purity carbon nanotubes. Diamond Relat. Mater., 2007, 16, 542549.

[37] Soria-Sanchez, M.; Maroto-Valiente, A.; Alvarez-Rodriguez, J.; Muñoz-Andres, V.; Rodriguez-Ramos, I.; Guerrero-Ruiz, A. Carbon nanostrutured materials as direct catalysts for phenol oxidation in aqueous phase. Appl. Catal. B- Environ., 2011, 104, 101-109.

[38] Mateos, J.M.J.; Fierro, J.L.G. X-ray photoelectron spectroscopic study of petroleum fuel cokes. Surf. Interf. Anal., 1996, 24, 223236.
[39] Cano-Serrano, E.; Blanco-Brieva, G.; Campos-Martin, J.M.; Fierro, J.L.G. Acid-functionalized amorphous silica by chemical grafting quantitative oxidation of thiol groups. Langmuir, 2003, 19(18), 7621-7627.

[40] Figueiredo, J.L.; Pereira, M.F.R.; Freitas, M.M.A.; Orfao, J.J.M. Modification of the surface chemistry of activated carbons. Carbon, 1999, 37, 1379-1389.

[41] Figueiredo, J.L.; Pereira, M.F.R.; Freitas, M.M.A.; Orfao, J.J.M. Characterization of active sites on carbon catalysts. Ind. Eng. Chem. Res., 2007, 46, 4110-4115.

[42] Gomes, H.T.;Miranda, S.M.; Sampaio, M.J.; Figueiredo, J.L.; Silva, A.M.T.; Faria, J.L. The role of activated carbons functionalized with thiol and sulfonic acid groups in catalytic wet peroxide oxidation. Appl. Catal. B-Environ., 2011, 106, 390-397.

[43] Terzyk, A.P. Further insights into the role of carbon surface functionalities in the mechanism of phenol adsorption. J. Colloid Interf. Sci., 2003, 268, 301-329.

[44] Castillejos, E.; Bachiller-Baeza, B.; Rodriguez-Ramos, I.; Guerrero-Ruiz A. An immersion calorimetry study of the interaction of organic compounds with carbon nanotube surfaces Carbon, 2012, 50, 2731-2740.

[45] Castillejos-Lopez, E.; Bachiller-Baeza, B.; Guerrero-Ruiz, A.; Rodriguez-Ramos, I. An immersion calorimetric study of the interactions between some organic molecules and functionalized carbon nanotube surface. Thermochim. Acta, 2013, 567, 107-111.

[46] Szymanski, G. S.; Rychlicki, G. Catalytic conversion of propan-2ol on carbon catalysts. Carbon, 1993, 31, 247-257.

[47] Chen, G.; Li, S.; Jiao, F.; Yuan, Q. Catalytic dehydration of bioethanol to ethylene over $\mathrm{TiO}_{2} / \mathrm{gamma}-\mathrm{Al}_{2} \mathrm{O}_{3}$ catalysts in microchannel reactors. Catal. Today, 2007, 125, 111-119.

[48] Varisli, D.; Dogu, T.; Dogu, G. Silicotungstic acid impregnated MCM-41-like mesoporous solid acid catalysts for dehydration of ethanol. Ind. Eng. Chem. Res., 2008, 47, 4071-4076.

[49] Zaki, T. Catalytic dehydration of ethanol using transition metal oxide catalysts. J. Colloid Interf. Sci., 2005, 284, 606-613.

(C) Almohalla et al.; Licensee Bentham Open.

This is an open access article licensed under the terms of the Creative Commons Attribution Non-Commercial License (http://creativecommons.org/licenses/ by-nc/3.0/) which permits unrestricted, non-commercial use, distribution and reproduction in any medium, provided the work is properly cited. 